Revue d'histoire de l'enfance « irrégulière »

Le Temps de l'histoire

1 | 1998

La protection de l'enfance : regards

Quelques éléments pour l'histoire du "Conseil technique de l'enfance déficiente et en danger moral" de 1943

Approche sociolinguistique et historique

Christian Rossignol

C OpenEdition

Journals

Édition électronique

URL : http://journals.openedition.org/rhei/11

DOI : $10.4000 /$ rhei. 11

ISBN : 978-2-7535-1638-0

ISSN : $1777-540 \mathrm{X}$

Éditeur

Presses universitaires de Rennes

Édition imprimée

Date de publication : 15 novembre 1998

Pagination : 21-39

ISSN : 1287-2431

Référence électronique

Christian Rossignol, « Quelques éléments pour l'histoire du "Conseil technique de l'enfance déficiente et en danger moral" de 1943 », Revue d'histoire de l'enfance «irrégulière » [En ligne], 1 | 1998, mis en ligne le 20 juin 2007, consulté le 03 décembre 2020. URL : http://journals.openedition.org/rhei/11 ; DOI : https://doi.org/10.4000/rhei.11 


\section{Quelques éléments pour l'histoire du "Conseil technique de l'enfance déficiente et en danger moral" de 1943 Approche sociolinguistique et historique}

\section{Introduction}

Lorsque j'ai entrepris d'aborder cette question du Conseil technique de 1943, j'ai sous-estimé l'ampleur des difficultés que j’allais rencontrer et la nature du problème auquel j'allais me trouver confronté.

Chercheur dans le domaine des sciences du langage, connaissant assez bien les secteurs professionnels concernés, mes lectures m'avaient permis d'acquérir une certaine connaissance de la période dont il est ici question et une claire conscience du fait qu'il nous manque des éléments essentiels pour comprendre ce qui s'est joué à ce moment de notre histoire.

Il m'a fallu d'abord constater que la plupart des chercheurs et des historiens qui ont rencontré ce problème se sont en quelque sorte dérobés devant l'obstacle; ils l'ont, plus ou moins consciemment, plus ou moins habilement, contourné et, finalement, le plus souvent, ils ont abandonné la partie.

Exception faite pour l'ouvrage de Michel Chauvière, ${ }^{(3)}$ les ouvrages de référence, déjà anciens, publiés par des universitaires, sociologues ou historiens de métier qui prennent pour objet d'étude La police des familles, ${ }^{(4)}$ Le travail social, ${ }^{(5)}$ Le métier d'éducateur, ${ }^{(6)}$ et qui développent à cette occasion des analyses souvent pénétrantes du fonctionnement institutionnel, ont pour caractéristique commune de "faire l'impasse" sur l'histoire de cette période.

L'ouvrage de Francine Muel-Dreyfus, Le métier d'éducateur, nous en fournit un exemple instructif sur lequel je m'arrêterai un instant. Elle affirme en introduction :

\section{Christian \\ Rossignol $^{22}$}

(1) Ce texte est la

communication de

l'auteur aux Journées

d'études L'Éducation sur-

veillée aurait eu 50 ans,

Vaucresson, les 9 et 10

novembre 1995.

(2) Chercheur au

CNRS.

(3) M. Chauvière,

Enfance inadaptée :

l'héritage de Vichy, Paris,

Éditions ouvrières, 1980

et $1987,315 \mathrm{p}$.

(4) J. Donzelot, $\mathrm{La}$ police des familles, Paris, Éditions de Minuit, 1977, 220 p.

(5) J. Verdès-Leroux, Le travail social, Paris, Éditions de Minuit, 1978, 273 p.

(6) F. Muel-Dreyfus, Le métier d'éducateur,

Paris, Éditions de Minuit, 1983, 269 p. 
(7) F. Muel-Dreyfus, op. cit., p. 9. C'est l'auteur qui souligne.

(8) F. Muel-Dreyfus, op. cit., p. 238.

(9) Par ce terme, traduit en français par “compulsion de répétition”, Freud désigne un processus incoercible par lequel un sujet se place activement dans des situations pénibles, répétant ainsi une expérience ancienne sans se souvenir du prototype et avec au contraire l'impression très vive qu'il s'agit de quelque chose qui est pleinement motivé dans l'actuel.
"On ne peut comprendre le présent par le présent. Et, en demandant à l’histoire de nous dire comment ont été produites au cours du temps des positions, on lui fait jouer le rôle d'une sorte de psychanalyse du monde social, susceptible d'éclairer le refoulé qui hante la vie institutionnelle. " ${ }^{(7)}$

La perspective paraît intéressante et nous pourrions nous attendre à ce qu'elle s'attache à dévoiler une partie de ce "refoulé qui hante la vie institutionnelle ». Or, l'ouvrage comporte effectivement un chapitre historique d'une soixantaine de pages, intéressant et bien documenté : "Chapitre 4 : La rééducation, un passé plein d'avenir " (p. 203-264). On y trouve un sous-chapitre intitulé "1920-1940: changement et continuité ", immédiatement suivi d'un autre intitulé "1945-1970 : L'irrésistible ascension de "l'inadaptation" ". Mais, de 1940 à 1945, que s'est-il passé? Francine Muel-Dreyfus évite de parler de la période 1940-1945 et mentionne simplement :

"Par la loi du 3 juillet 1944, considérée comme une "charte de l'enfance malbeureuse", le gouvernement de Vichy laissait un cadre législatif permettant (une) réorganisation; or, bien que de nombreuses décisions prises dans ce domaine pendant l'occupation aient été reconduites, ce cadre est annulé en 1945. " ${ }^{(8)}$

Nous n'en saurons pas plus en lisant cet ouvrage, mais il peut être intéressant de remarquer que les titres des chapitres qui entourent ce " trou de mémoire " n'ont pas une valeur purement dénotative : ce sont des figures de rhétorique. Un psychanalyste ou un sémiologue remarquerait sans doute que "un passé plein d'avenir " ou "l'irrésistible ascension de l'inadaptation " constituent des traits d'esprit qui, comme tels, expriment par un déplacement une idée qui ne peut être formulée autrement.

- La première, le passé plein d'avenir, évoque avec une ironie amère l'inéluctable fatalité de la répétition dans l'histoire de ce secteur professionnel et le caractère incoercible du "Wiederholungszwang". (9)

- La seconde est une allusion évidente à l'œuvre de B. Brecht, La résistible ascension d'Arturo Ui, qui, en 1941, évoque la montée en puissance d'un système totalitaire.

Le mot d'esprit, disait Freud, est comme "une allusion", "un déguisement ", " une traduction dans un autre langage ". Il se présente ici comme un compromis entre une volonté de dire quelque chose et d'excellents 
motifs de ne pas le faire, car nous savons bien que ce n'est pas sans s'exposer à quelques désagréments que l'on tente de mobiliser des souvenirs dans ce domaine.

Le caractère plaisant du mot d'esprit permet de faire l'économie de l'effort nécessaire pour surmonter les obstacles qui s'opposent à la «levée du refoulement $"$ Ou, pour dire les choses plus simplement, il permet de faire l'économie de l'effort qui serait nécessaire pour concevoir et parler clairement.

Mais ne croyez pas que, par ces remarques, mon intention soit de critiquer ou de montrer du doigt les auteurs que je viens de citer. Cette critique, si elle devait être développée, s'appliquerait tout aussi bien à mon propre travail.

J'ai, en effet, il y a quelques années, entrepris la rédaction d'un ouvrage intitulé Le poids de l'bistoire et les orientations actuelles des politiques sociales, ouvrage dont la rédaction s'est trouvée interrompue. Mais lorsque, pour préparer cette intervention, j’ai essuyé la poussière qui recouvrait le manuscrit, je me suis aperçu que le dernier chapitre rédigé s'intitule "L'enfance malheureuse : juin 1940-avril 1942 »; le chapitre suivant, qui devait s'intituler "Le basculement politique du printemps 1942 et l'invention de l'enfance inadaptée ", n'a jamais été rédigé. Je pourrais évoquer les multiples "bonnes raisons " qui m'ont conduit à interrompre ce travail, mais, à m'en tenir à la rigueur des faits, il me faut constater qu'en fin de compte je n'ai pas agi différemment des auteurs que je viens de citer.

Aujourd'hui, il me faut dire quelque chose sur ce sujet.

J'essaierai donc d'éviter, autant que faire se peut, les jeux de mots, les euphémismes et les figures de rhétorique. Je m'attacherai par contre à mettre l'accent sur ce que nous ignorons de ce qui s'est passé à ce moment de notre histoire, plutôt que sur ce que nous en savons ou croyons en savoir à travers des reconstructions autojustificatives ou «en trompe l'ail ». 


\section{LES PREMIÈRES FORMULATIONS DU PROBLÈME}

Dans les dernières années de la IIIème République, les établissements et services qui prennent en charge les problèmes désignés à l'époque par des expressions telles que "enfance malheureuse ", "en danger moral ", " anormale ", "déficiente ", "délinquante ", etc., forment un puzzle d'institutions prises dans un jeu complexe de rivalités, d'alliances et d'oppositions, mais le plus souvent jalouses de leurs prérogatives et de leur autonomie. Existent alors notamment :

- un secteur privé, le plus souvent créé et géré par des congrégations religieuses généralement dépourvues de moyens et en opposition avec l'État : orphelinats, "refuges", "Bons Pasteurs", etc.;

- quelques classes et écoles de perfectionnement instituées par la loi de 1909, sous tutelle du ministère de l'Instruction publique, qui restent très peu nombreuses;

- quelques "maisons d'éducation surveillée" et "maisons de correction" sous tutelle de l'Administration pénitentiaire; elles sont dans une situation si détériorée qu'elles font l'objet de campagnes de presse qui les désignent comme "bagnes d'enfants";

- des "instituts médico-pédagogiques" installés dans l'enceinte des asiles d'aliénés, où la misère et la promiscuité ne sont pas moindres.

Mais, il n'existe à cette époque ni structure de coordination entre ces services, ni politique sociale globale, ni contrôle de l’État.

Autrement dit, il existe bien évidemment, comme à toutes les époques, des enfants confrontés à des problèmes et à des situations de tous ordres et diverses solutions institutionnelles sont en place pour leur venir en aide. Mais ces enfants ne constituent pas un ensemble définissable susceptible d'être désigné par un terme unique pouvant induire l'idée qu'ils ont entre eux quelque chose de commun. Et il n'existe pas non plus un "secteur professionnel" tel que nous le concevons aujourd'hui, appelé à prendre en charge un problème ainsi globalisé.

Ce qui sera ultérieurement désigné du terme générique "enfance inadaptée " ne constitue pas encore ce que les sémiologues appelleraient 
une "unité culturelle"; cela ne constitue pas encore une entité concevable, du fait qu'aucune conception normative, théorique, juridique ou idéologique ne rassemble les faits concernés sous une même notion, ni n'est parvenue à en imposer une interprétation unifiée et dominante.

Certes, le projet est bien "dans l'air du temps" et une jeune discipline en plein développement a déjà des prétentions à réaliser cette unité.

Dès 1914, Georges Heuyer, fondateur de la neuropsychiatrie infantile, posait la question suivante : "Qu'y a-t-il de commun entre les enfants anormaux de Bicêtre, les délinquants de la Petite Roquette et les élèves des classes de perfectionnement?». Bien entendu, il ne posait cette question que pour introduire une réponse déjà prête : "le même aspect, la même hérédité, la même origine familiale ». ${ }^{(10)}$

Ce faisant, il indique bien, du même coup, quels sont les acteurs institutionnels et les obstacles qui s'opposent encore à ce que la neuropsychiatrie infantile puisse jouer le rôle fédérateur auquel elle aspire.

Ces obstacles ne seront levés qu'au terme d'un processus assez complexe qui se déroule dans le courant de la seconde guerre mondiale et aussitôt après. La description de ce processus demanderait du temps. Je me contenterai ici d'en situer les principales étapes.

\section{LE CONTEXTE POLITIQUE}

Dans le courant des années 30, plusieurs projets de coordination des œuvres concernées par la protection de l'enfance seront élaborés. ${ }^{(11)}$ Mais, dans le contexte particulier de la IIIème République, où les gouvernements sont paralysés par l'équilibre des intérêts antagonistes, aucune réforme d'ensemble n'est envisageable, et cette situation n'est pas propre aux institutions de l'enfance. Dans ce contexte, aucune réforme, même minime ou relevant d'un simple bon sens technique, ne peut aboutir.

Après la défaite de juin 1940 et la remise du pouvoir entre les mains du Maréchal Pétain, des réformateurs de tous horizons, qui avaient rongé leur frein sous la IIIème République, caressent l'espoir de pouvoir enfin mener à bien leurs projets. Bon nombre d'entre eux, d'ailleurs, commettent l'erreur de croire qu'il est possible d'entreprendre une
(10) G. Heuyer,

Enfants anormaux et délinquants juvéniles, nécessité de l'examen psychiatrique des écoliers, Paris, 1914. Cité par Michel Chauvière.

(11) Cf. M. Chauvière, op. cit., p. 25 à 28. 
(12) R.O. Paxton, Vichy France, Old Guard and New Order, 19401944. Traduction française La France de Vichy 1940-1944, Paris, Seuil, 1973.

(13) Darlan, cité in H. Du Moulin de la Barthète, Le temps des illusions. Souvenirs, juillet 1940-avril 1942, Genève, Le Cheval ailé, 1946, p. 347. réforme durable et profonde des institutions dans un pays occupé. Cet espoir et cette illusion sont d'autant plus forts que le Maréchal Pétain n'est pas l'homme de l'extrême droite, mais le représentant d'un consensus national. Ses premiers gouvernements ne comportent aucun représentant de l'extrême droite pro-nazie, et ceux-ci n'y accéderont qu'après le 15 avril 1942 et le retour de Laval au pouvoir imposé par l'occupant.

Pour comprendre ce qui a pu se passer, il faut, je crois, garder à l'esprit qu'il n'y a pas eu "un" gouvernement de Vichy; il y en a eu au moins six, et ceux-ci étaient loin d'être politiquement homogènes. Ils étaient traversés par des courants politiques et philosophiques souvent très différents et dont le seul ciment semble avoir été leur anti-bolchevisme. Ces courants, à tour de rôle, se sont trouvés en position dominante.

Les historiens ont coutume de distinguer trois périodes principales. Nous distinguerons :

- une première période qui s'étend de l'armistice au 13 décembre 1940. Les gouvernements y sont dominés par un courant traditionaliste, partisan de la restauration d'un ordre moral catholique et d'un retour aux valeurs traditionnelles. Le Maréchal et ses principaux conseillers appartiennent incontestablement à ce courant que Paxton ${ }^{(12)}$ appelle «le Vichy de la biérarchie et de l'autorité ". Ce courant, à l'apogée de son influence en 1940, ne cessera par la suite de perdre du terrain, et ses membres, à la Libération, paieront le plus lourd tribut à "l'épuration".

- une seconde période, qui débute avec l'éviction de Laval le 13 décembre 1940, s'achève avec son retour le 15 avril 1942. Les technocrates de l'Amiral Darlan occupent les postes clefs et sont, dit ce dernier, «chargés de remplacer les puceaux de sacristie [...], les généraux, les séminaristes qui ont échoué dans la conduite des affaires françaises ". ${ }^{(13)}$ Ce sont eux que Paxton appelle "le Vichy de la rationalisation et de l'expansion industrielle». - une troisième période débute en avril 1942 avec le retour de Laval, imposé par les Allemands, et s'achèvera avec la Libération. Elle est marquée par l'éviction définitive des traditionalistes et l'entrée au gouvernement de l'extrême droite pro-nazie.

Dans ces contextes, plusieurs projets de coordination, plusieurs plans d'action en faveur de l'enfance ont pu voir le jour. Je me limiterai à évoquer 
les deux principaux, ayant une portée nationale. Le premier, que j'appelle-

(14) P. Pétain, déclaration du 25 juin 1940. seul, ni peut-être le principal promoteur, se développe entre octobre 1940 et le début de l'année 1942. Le second est celui qui sera issu des travaux du "Conseil technique de l'enfance déficiente et en danger moral" de 1943.

À première vue, les deux plans pourraient paraître se situer dans une certaine continuité. Bien entendu, le second s'appuie sur les réalisations concrètes du premier, mais en y regardant de plus près, nous nous apercevrons que, sur le plan des idées, le second marque une nette rupture par rapport au premier. Ils s'opposent en ce qui concerne :

- leurs commanditaires et les partenaires institutionnels qui ne sont pas les mêmes;

- la géographie de leurs implantations dont le centre de gravité se situe entre Toulouse et Vichy pour le premier, à Paris pour le second;

- les personnalités qui les ont soutenus qui n'appartiennent manifestement pas à la même famille politique;

- les philosophies sociales qui les sous-tendent et qui sont, sur bien des points, radicalement opposées, notamment en ce qui concerne le rôle et les droits respectifs des familles et de l'État;

- leurs objectifs et leurs véritables finalités qui étaient peut-être très différentes, mais cela demeure une zone d'ombre qui restera à explorer.

\section{LE PLAN PLAQUEVENT}

Pour le Maréchal, "le redressement intellectuel et moral de la jeunesse " ${ }^{(14)}$ occupe une place centrale dans la doctrine de la "Révolution nationale » et constitue pour lui l'assise sur laquelle elle devrait s'établir.

Dans le contexte de son arrivée au pouvoir, la question acquiert une dimension nouvelle alors que le chômage sévit dans un pays désorganisé par l'exode et que de nombreuses familles de prisonniers sont dans une situation précaire. Le problème est encore aggravé par le fait que 90.000 jeunes de la classe 1940, démobilisés et livrés à eux-mêmes sur les routes ou sur le pavé des villes, risquent de glisser vers la délinquance et font l'objet de convoitises de la part des organisations nazies. 
(15) Jean Plaquevent (1901-1965), aumônier du Bon Pasteur de Pau en 1929, fondera l’École de rééducateurs de Toulouse en 1942.
En Octobre 1940, la direction de la Famille du ministère de l'Intérieur (Louis Lafont) demande à l'Abbé Jean Plaquevent ${ }^{(15)}$ de lui faire parvenir :

- une documentation sur "la recrudescence actuelle de la délinquance juvénile »;

- une aide pour "le recrutement des premiers cadres et la prospection de maisons susceptibles d'être acquises et aménagées pour les premiers centres de rééducation ";

- tous renseignements sur "les conditions psychopédagogiques et morales de la rééducation";

- des suggestions concernant un "plan d'action immédiate pour le sauvetage et la protection de l'enfance en danger moral ".

Ce "plan d'action immédiat "sera élaboré dans les jours qui suivent et il semble que Lafont ait demandé à Plaquevent de s'engager d'urgence dans sa mise en œuvre, en lui promettant que des moyens allaient lui être accordés.

Le 26 novembre 1940, l'intendance n'ayant pas suivi, dans une lettre adressée à M. Lafont, directeur de la Famille, les relations sont tendues et Plaquevent menace Lafont de s'adresser directement " à la personne même du Maréchal ». Ce qu'il fera d'ailleurs en février 1941, puis à nouveau le 26 mars 1941. Dès lors, les choses vont aller très vite.

Le 4 avril, son plan est accepté par Jacques Chevalier, secrétariat à la Famille et à la Santé. Acceptation assortie de l'ouverture d'un crédit de neuf millions de francs.

Dans une lettre du 29 mai 1941, le même Jacques Chevalier définit les grande lignes du dispositif à mettre en place et prévoit que le financement en sera assuré par le Secours national.

En novembre 1941, un rapport dont j'ignore l'auteur, adressé à "Monsieur le ministre à l'Éducation nationale et à la jeunesse " (Jérôme Carcopino), présente un projet d'arrêté portant création d'un "Comité interministériel pour la protection de la jeunesse en danger moral». Le plan d'action qui y est annexé, et que je ne détaillerai pas, prévoit notamment de " nommer pour une durée de quatre mois à compter du 1er novembre l'Abbé Plaquevent, conseiller technique près du directeur de la Formation des jeunes. Cette nomination permettrait de s'attacher le concours temporaire de ce spécialiste qui en même temps a su réaliser. (La demande de nomination a été adressée par mes soins à Monsieur Lamirand et à Monsieur Garrone, pour vous être soumise) ". 
On y apprend également que, sur intervention du Maréchal Pétain, une somme de cinquante millions de francs sur les fonds de la Loterie nationale est mise à la disposition du secrétariat général à la Famille.

Je ne pourrai, faute de temps, donner plus de détails sur ce "Plan Plaquevent", mais il importe de situer quelles sont les personnalités et les forces politiques qui le soutiennent. Pour cela un moyen rapide consiste à examiner les biographies des personnes impliquées dans sa mise en œuvre : vous verrez que cela dessine un "paysage politique" bien différent de celui dans lequel s'insère le Conseil technique de 1943.

PAUL BAUDOUIN est sorti major de Polytechnique en 1914. Ancien directeur général de la Banque d'Indochine, c'est un anglophile pacifiste, artisan de l'Armistice, mais néanmoins favorable au départ du Maréchal pour Alger et partisan de préparer progressivement le retour de la France dans la guerre aux côtés des Anglo-saxons. Le 17 juin 1940 il obtient, grâce à l'appui du général Weygand, le poste de ministre des Affaires étrangères dont rêvait Laval. Paul Baudouin s'intéresse de près aux problèmes de la jeunesse et s'est acquis une notoriété dans les milieux intellectuels chrétiens en publiant, en 1939, dans La revue des jeunes dirigée par Robert Garic, un manifeste intitulé "Discours à des jeunes qui entrent dans la vie ». Il semble que ce soit lui qui ait poussé le Maréchal à créer une structure ministérielle spécifiquement consacrée aux problèmes de la jeunesse. Le 9 novembre 1940, il déclare à Lyon :

"La jeunesse n'aura pas un uniforme unique, une idéologie unique; (chaque mouvement) pourra conserver son inspiration idéologique et ses méthodes d'action. Nous leur demandons une seule chose à tous : concentrer leur action vers une même voie, un même point de rapprochement fondamental, la Révolution nationale ».

JACQUES CHEVALIER est né à l'orée de la forêt de Tronçais, d'un père général du Génie ayant servi sous les ordres du Maréchal Pétain. Destiné très tôt à la carrière militaire, il refuse le moment venu d'embrasser la carrière des armes, car il souhaite devenir philosophe. Contre l'avis de son père, il entre à l'École normale supérieure, où il est reçu second à l'agrégation de philosophie, et bénéficie d'une bourse pour 
(16) P. Giolitto,

Histoire de la jeunesse sous Vichy, Paris, Librairie académique Perrin, 1991. aller étudier à Oxford. Filleul et ami du Maréchal avant d'en devenir ministre, il est doyen de la Faculté des lettres de Grenoble lorsque celuici lui propose, le 6 septembre 1940, le poste de secrétaire général, puis de secrétaire d'État à l'Instruction publique, aux Beaux-arts et à la Jeunesse. Il y restera jusqu'à l'arrivée de Darlan au pouvoir. Il sera alors remplacé par Jérôme Carcopino et nommé ministre de la Famille et de la Santé publique. En août 1941, il remet sa démission au Maréchal et retourne à son poste de doyen de la Faculté de Grenoble.

Sur le plan diplomatique, il est l'homme des contacts secrets avec l'Angleterre par l'intermédiaire de lord Halifax, son camarade d'Oxford, via les ambassades à Madrid. ${ }^{(16)}$

Sur le plan intellectuel, il est décrit par Pierre Giolitto comme "un personnage pur et dur. Un grand cbrétien et un homme de baute culture...". Disciple de Bergson et maître d'Emmanuel Mounier, auteur de nombreux ouvrages, il est considéré comme l'un des rénovateurs de la philosophie spiritualiste en France. Néo-thomiste, il insiste particulièrement sur la défense des droits des personnes face aux pouvoirs de l'État.

Pour mieux situer le personnage, nous pouvons dire aussi que c'est lui qui tient tête aux Allemands et refuse d'introduire le numerus clausus pour les enfants juifs dans les lycées et collèges.

Il leur tient tête également lorsqu'il interdit à l'école tout port d'insigne indiquant l'appartenance à une quelconque organisation, y compris les organisations pétainistes.

C'est lui encore qui, en décembre 1940, refuse de livrer le personnel enseignant d'Alsace et de Lorraine aux Allemands qui le réclament.

En 1941, à la mort de Bergson, il n’hésite pas à l'honorer publiquement, bien qu'il soit juif, ce qui est perçu à Paris comme une véritable provocation.

De retour à Grenoble, il facilite l'attribution de faux papiers à des étudiants désignés pour le STO et protège les étudiants juifs en leur permettant de s'inscrire en faculté au delà du contingent légal.

Mais son anticommunisme intransigeant lui jouera de mauvais tours à l'approche de la Libération. 
Parmi les personnalités qui soutiennent ce projet, citons également GEORGES LAMIRAND. Fils d'un inspecteur général de l'Instruction publique, licencié en droit et ingénieur de l'École centrale, il a fait une brillante carrière dans l'industrie. Passionné par les problèmes sociaux et chrétien fervent, il se plait à rappeler que l'usine qu'il dirigeait avant la guerre a abrité la première section de la JOC de France. Il se situe parfaitement dans l'orthodoxie de la première Révolution nationale, sauf en ce qui concerne la collaboration avec l'Allemagne. On peut noter à ce sujet que c'est lui, alors secrétaire d'État à la Jeunesse, qui accompagné du commandant PIERRE DUNOYER DE SEGONZAC, directeur de l'École nationale des cadres d'Uriage, tente en vain, le 9 novembre 1942, à la veille du débarquement allié en Afrique du Nord, de convaincre le Maréchal de prendre la tête de la lutte contre l'occupant, après avoir préalablement organisé son départ pour Alger. Il est la "bête noire" des milieux collaborationnistes qui lui reprochent notamment de s'opposer à la mise en place d'une jeunesse unique de type totalitaire.

Il est en effet partisan d'une organisation pluraliste et libérale des mouvements de jeunesse. Et il est important de noter que c'est une certaine conception des rôles respectifs de la famille et de l'État dans l'éducation qui constitue la ligne de partage entre les "maréchalistes" et les partisans d'une "jeunesse unique, prise en main par l'État, éduquée, disciplinée par l'État et uniquement par lui ", ${ }^{(1)}$ en vue de créer cette "jeunesse intrépide et cruelle » dont parle Hitler dans Mein Kampf.

Il faudrait encore citer LOUIS GARONNE, qui est un pur produit de l'École des Roches, où il enseigne la philosophie. Nommé, en janvier 1941, directeur de la Jeunesse puis directeur de la Formation des jeunes par Jacques Chevalier, il est lui aussi chrétien, passionné par les problèmes sociaux et honni par les collaborationnistes. Il est à noter que c'est également lui qui a encouragé et aidé Pierre Dunoyer de Segonzac à créer l'École des cadres d'Uriage.

À la fin de l'année 1941, le Secrétariat général à la Jeunesse, aux destinées duquel ont présidé successivement Paul Baudouin, puis Jérôme Carcopino, 
(18) Ce qui ne l'empêchera pas, au moment opportun, d'adhérer au parti communiste. ministres de l'Éducation nationale, est encore une puissante machine administrative, richement dotée, dominée par des hommes de haute culture, chrétiens, anti-collaborationnistes et résolument anti-communistes.

\section{AVRIL 1942 : LA DÉRIVE FASCISANTE DU SECRÉTARIAT GÉNÉRAL À LA JEUNESSE}

Jusqu'à la fin de l'année 1941, les hommes qui composent l'équipe de Lamirand restent en mesure d'imposer leurs orientations au SGJ. Mais, à partir d'avril 1942, avec le retour de Laval au pouvoir et le remplacement de Jérôme Carcopino par Abel Bonnard, surnommé "Gestapette" à cause de ses options politiques et de ses préférences sexuelles, commence ce que Giolitto appelle "la dérive fascisante du SGJ". Le "chef historique" est conservé pour un temps, mais ses plus proches collaborateurs sont progressivement remplacés par des hommes d'Abel Bonnard. Louis Garonne, bras droit de Lamirand, est écarté et sera remplacé, le 9 juin 1941, par Georges Pelorson, précédemment directeur de la propagande des jeunes en zone occupée et fervent partisan d'une jeunesse unique. Ses adversaires l'ont surnommé "Baldur von Pelorson", vraisemblablement par référence à Baldur von Schirach, reich-jugendführer de la Hitlerjugend. Jacques Bousquet, directeur de l'École des cadres de la Chapelle-en-Serval, devenue grâce à lui un haut lieu de la propagande nazie, s'est illustré en exécutant le salut hitlérien devant la commission d'études des questions de la Jeunesse; ${ }^{(18)}$ il est nommé inspecteur général de la Jeunesse.

Ces hommes utilisent toujours la Révolution nationale comme référence, mais, s'ils gardent l'expression, ils en changent le contenu; il ne s'agit plus dès lors d'offrir à la jeunesse « des vieilleries poussiéreuses sentant la naphtaline et l'encens". Il ne s'agit plus, écrit Je suis partout, d'une Révolution nationale française, mais d'une "Révolution nationale et sociale européenne ».

Le projet politique du gouvernement est fondé sur l'hypothèse d'une victoire de l'Allemagne et défini comme un projet d'intégration européenne; c'est le projet Laval d'" une Europe unifiée sous l'égide de l'Allemagne, où la France pourrait jouer le rôle d'un brillant second ». 
Ainsi, à compter du retour de Laval, les collaborateurs pro-nazis marquent des points, mais s'ils parviennent à détruire l'œuvre de leurs adversaires, ils ne parviennent pas pour autant à imposer leur modèle. Pratiquement vidé de sa substance, le SGJ sera supprimé le 31 décembre 1943 et remplacé par un simple Commissariat général ayant à sa tête Maurice Gait, directeur de cabinet d'Abel Bonnard, qui se bornera à organiser les départs pour le STO.

À la fin de l'année 1941, faute de documents d'archives, et probablement aussi d'un travail suffisant, je perds la trace de ce que j'ai appelé le "Plan Plaquevent" jusqu'à l'ouverture, en 1943, des travaux du "Conseil technique de l'enfance déficiente ou en danger moral", dont la mission sera d'élaborer un nouveau projet appelé à le remplacer.

\section{LE “CONSEIL TECHNIQUE DE L'ENFANCE DÉFICIENTE OU EN DANGER MORAL"}

Ce projet voit le jour dans un contexte et selon des modalités très différents du précédent; il ne s'agit plus d'un projet élaboré à partir d'une pratique de terrain, pour répondre aux problèmes concrets et urgents de l'enfance et de la jeunesse au lendemain de la défaite. C'est un projet politique décidé au plus haut niveau, qui semble venir directement de la présidence du Conseil ou tout au moins avoir transité par lui.

C'est une mission gouvernementale qui n'est plus comme précédemment placée sous l'autorité du ministre de l'Éducation, mais directement commanditée par Pierre Laval et placée sous le patronage du ministre de la Santé et de la Famille, le Dr Grasset, fidèle parmi les fidèles de Laval et qui, sauf erreur de ma part, a dû remplacer l'Amiral Platon, fidèle du Maréchal.

Le ministre Grasset qui assiste aux séances déclare «qu'il soubaite qu'on puisse mettre sur pieds, lors de son passage au gouvernement, d'une façon définitive, toute la question de l'enfance déficiente ou en danger moral qui a été trop souvent abandonnée par les pouvoirs publics dans des conditions navrantes ". ${ }^{(19)}$

Le Conseil est présidé par le Docteur Heuyer, fondateur de la neuropsychiatrie infantile. ${ }^{(20)}$ Après avoir évoqué les travaux et projets précédents il déclarera : 
(21) Procès-verbal de la réunion du Conseil technique des 26 et 27 juin 1944.

(22) G. Heuyer, "Le Conseil technique, son fonctionnement, ses travaux", in La santé de l'homme, $\mathrm{n}^{\circ} 27$, janvier 1945

(23) La phrase placée en exergue à la publication des travaux est intéressante :

"Le médecin de l'avenir sera d'abord un éducateur et l'instituteur sera son principal collaborateur ».

(24) Au même moment, en Allemagne, " des médecins ayant reçu une formation anthropologique et psychiatrique avaient obtenu, le 9 mars 1943, le droit et le devoir de mener la sélection et la mise à mort des victimes; ils se battirent pour les garder [...] ». "Auschwitz et les établissements d'extermination psychiatrique se valaient. Les médecins y dirigeaient la sélection et la mise à mort. Ils en avaient
"À la suite des changements de cabinet, ces projets n'ont pu voir le jour. Toute la question de l'enfance déficiente et en danger moral a été reprise en 1943, au moment de la création du Service de coordination. " ${ }^{(21)}$

Ceci étant dit, c'est là que les choses deviennent difficiles; les travaux manquent, les archives sont rares et difficiles à interpréter. Je dispose en tout et pour tout des comptes rendus de cinq réunions plénières qui viennent des archives de l'abbé Plaquevent. Tous les autres documents, y compris la synthèse des travaux par Heuyer, sont des documents publiés après la Libération et qui ne dissimulent pas toujours très bien leur caractère autojustificatif.

Cela n'empêche pas, et même impose, je crois, de se poser des questions, même si celles-ci doivent rester encore un certain temps sans réponse. Ainsi, par exemple, lorsque Heuyer définit les missions du "Conseil technique" :

"Lorsque le Conseil technique a été créé, par arrêté du 25 juillet 1943, nous avons pensé qu'il avait pour but d'établir le statut de l'enfance déficiente et en danger moral et de fournir, dans toutes les circonstances où l'enfant a besoin d'être assisté, les techniques et les méthodes qui permettent d'assurer son dépistage, son observation et son reclassement dans la vie sociale. " ${ }^{(2)}$

Il est permis de penser que ces objectifs imprécisément définis au départ n'étaient pas forcément les mêmes pour tous au sein du Conseil technique et, en particulier, qu'ils n'étaient pas les mêmes que ceux des commanditaires du projet au sein du gouvernement Laval.

Incontestablement, fait radicalement nouveau, les médecins, comme l'a pointé $\mathrm{M}$. Chauvière, dominent largement ce Conseil technique; les neuropsychiatres en particulier y sont sur-représentés et trouvent là une occasion inespérée de réaliser leur rêve. ${ }^{(23)}$ Mais qu'attendait-on d'eux en échange? Qu'attendait ce gouvernement, omniprésent tout au long des travaux, en échange d'un tel cadeau? ${ }^{(24)}$

Les médecins sont même de plus en plus sur-représentés au fil du temps; dans aucun des comptes rendus dont je dispose, l'Abbé Plaquevent, bien qu'officiellement membre de ce Conseil, ne figure dans la liste des présents et une seule fois il y est représenté par le Dr Mathis. Dans les dernières séances du Conseil (juin 1944), il n’y a plus aucun repré- 
sentant de l'Éducation nationale; la dernière à y avoir participé, Mlle Mezeix, s'est fait excuser sous un prétexte qui ne fait illusion pour personne. Ce qui semble beaucoup contrarier Heuyer, d'autant qu'elle a refusé d'accorder sa signature au rapport de la commission à laquelle elle participe.

En ce qui concerne le contenu des travaux, il est impossible d'en rendre compte ici dans le détail, mais nous pouvons dire qu'ils s'organisent prioritairement autour de trois axes :

- création d'une nomenclature et classification de ceux qui seront désormais appelés des "inadaptés";

- nomenclature des types d'établissements;

- nomenclature des personnels de la "rééducation".

Autrement dit, c'est de la structuration d'un champ professionnel nouveau qu'il s'agit.

Mise en place sur l'ensemble du territoire national d'un redoutable dispositif de "dépistage" fondé sur des services sociaux unifiés concernés tant par "l'enfance déficiente et en danger moral que (par) l'enfance délinquante ": les "Offices publics d'bygiène sociale " sont destinés à alimenter des "Centres d'observation et de triage".

Cet ensemble devant en outre être contrôlé par un "Conseil de protection de l'enfance" dont les fonctions sont ainsi définies par le procureur Jean Chazal :

"J'ajouterai qu'il est dans mon intention de faire contrôler ce service social de protection de l'enfance par le "Conseil de protection de l'enfance" ".

"Ce conseil aura une mission de contrôle et une mission de décision, c'est-àdire qu'il devra placer dans les établissements de protection de l'enfance l'ensemble des enfants dont les familles n'acceptent pas le placement volontaire, enfants qui par ailleurs ne relèvent pas de l'autorité judiciaire ". ${ }^{(25)}$

Rien, par contre, ou très peu de chose concernant les techniques ou les méthodes de rééducation dans les comptes rendus des réunions du Conseil technique. obtenu le droit, et n'étaient pas disposés à se le laisser ravir par d'autres. " Höss, Kommandant in Auschwitz. Trad. française : Le commandant d'Auschwitz parle, Paris, Julliard, 1949. Cité par Mûller Hill B., in Tödliche Wissenschaft. Trad. française : Science nazie, science de mort, Paris, Odile Jacob, 1989.

(25) J. Chazal, Introduction de la réunion du 11 janvier 1944. 
(26) Procès-verbal de la réunion du 30 mars 1944 au ministère de la Santé, p. 10.

(27) D. Lagache, 1943, "Nomenclature et classification des jeunes inadaptés”, Sauvegarde, $n^{\circ} 2,3,4,1946$.

Cf. note 1 .

(28) Trois ans auparavant, le Professeur Von Verchuer, directeur de l'IEG d'anthropologie écrivait : "pour la protection concrète du patrimoine béréditaire et de la race, le plus important est de distinguer ceux qu'il faut éliminer de ceux qu'il faut promouvoir. Il serait souhaitable que de tels fichiers soient dressés dans le Reich tout entier pour que l'inadaptation sociale soit combattue par tous les moyens dons nous disposons. " Der Erbarzt, 1940, 8, 235. [C'est moi qui souligne.] (29) Au même moment, en 1943, en Allemagne, après que les programmes d'extermination des tziganes et

\section{LE POIDS DES MOTS}

Si nous ne disposons que de peu de documents originaux, il reste que les questions de choix terminologiques occupent une place importante, tant dans les travaux du conseil que dans les discours autojustificatifs postérieurs à la Libération. Si nous les interprétons en fonction du contexte politique et des circonstances que nous commençons à bien connaître, ils sont susceptibles de nous éclairer sur bien des points.

La première commission du Conseil technique se consacre à la "standardisation de la nomenclature des jeunes inadaptés", avec notamment pour objectif "d'établir des définitions de valeur internationale". Une "nomenclature et classification des jeunes inadaptés" est présentée par le professeur Daniel Lagache lors de la séance du 11 janvier 1944.

Cette nomenclature est composée de deux classifications, mais nous verrons qu'il s'agit en fait d'une classification à double entrée.

La première classification, dite "médico-psychologique", est établie en se plaçant "à un point de vue aussi descriptif que possible, en évitant de faire intervenir les théories et les doctrines; c'est-à-dire que la symptomatologie doit jouer un rôle plus important que l'étiologie et la pathogénie."

Autrement dit, cette classification ne prend en considération ni les causes des troubles, ni ce qui peut déterminer leur évolution, et transforme par là-même les sujets en souffrance de l'abbé Plaquevent en individus sans histoire.

Dans le débat qui suit, Lagache estime que "s'il devait suivre $M M$. Dechaume et Girard dans leur projet de classification, il faudrait multiplier alors les rubriques selon les réactions, la personnalité, le pronostic, les indications thérapeutiques etc., $[. .$.$] mais cette dernière solution n'a peut-être pas une grande$ utilité pratique ». ${ }^{(26)}$

Question pratique, pour Lagache, il s'agit de déterminer comment il convient de "traiter" ces enfants, ce qu'il convient d'en faire.

Ce sera l'objet de la seconde classification dite "médico-pédagogique", établie en se plaçant cette fois-ci, explique Lagache, " $d u$ point de vue de l'assistance et du traitement dont ces enfants relèvent". 
Le principe sur lequel repose cette seconde classification est clairement énoncé :

"Dans l'ensemble on peut distinguer les récupérables, les semi-récupérables et les non-récupérables. À cette progression se superposent approximativement les degrés de la classification ci-dessous. " ${ }^{(27)}$

Le Docteur Dublineau proposait quant à lui de "séparer nettement adaptables, semi-adaptables et inadaptables".

Nous ne pouvons éviter la question de ce que pouvait signifier et impliquer en 1943, au plus fort de la mise en œuvre de la "solution finale", le fait d'être classé "inadaptable" ${ }^{(28)}$ ou "irrécupérable" ?

De plus, lorsque le compte rendu nous apprend que la solution préconisée pour les "irrécupérables " est une "section d'anormaux des hôpitaux psychiatriques", on peut se poser la question de savoir si ces psychiatres ignoraient ce qui se passait à ce moment, en France et en Allemagne dans les hôpitaux psychiatriques? C'est peu vraisemblable.

La première initiative de cette commission de la nomenclature sera, suite à un débat, d'inventer une nouvelle dénomination, un terme générique qui subsume et remplace ceux déjà utilisés, une appellation nouvelle destinée à globaliser le problème et à désigner l'ensemble des enfants concernés.

Deux notions sont en concurrence

- la notion d' "enfance irrégulière ", qui ancre le problème dans sa dimension de rapport à la norme, c'est-à-dire dans sa dimension culturelle;

- la notion d' "enfance inadaptée ", qui ancre le problème dans une dimension biologique.

Cette opposition caractérise bien le clivage idéologique entre les deux versions de la Révolution nationale. En 1943, c'est bien entendu la notion d'enfance inadaptée qui devait être adoptée.

Mais, pour mesurer le poids de cette décision, il ne faut pas perdre de vue le contexte. Il s'agit du regroupement sous une même notion empruntée à la biologie de la fin du XIXème siècle - récupérée depuis quelque temps déjà par l'idéologie nazie - ${ }^{(29)}$ de réalités très hétérogènes précédemment désignées par les termes d' "enfance malheureuse ", " anormale ", "déficiente ", "en danger moral » ou " abandonnée ", en passant par des malades mentaux eurent été menés à bien, le ministère de

l'Intérieur et la Deutsche Forschungsgemeinschaft (DFG, Société d'assistance de la science allemande) mettent en place un nouveau programme nommé « Évaluation spéculative des jeunes asociaux". Ce programme prévoyait l'examen "crimino-biologique" des jeunes asociaux par des psychiatres, l'objectif étant de distinguer entre ceux qu'il fallait mettre à mort et ceux qui pouvaient être considérés comme récupérables.

Le Professeur Heyde, expert en psychiatrie, écrit le 24 avril 1942 au ministre du Reich de la Science, de l'éducation et de la formation populaire qu'il « est nécessaire de détecter les types de personnalités incapables d'emblée, par nature, de recevoir quelque éducation que ce soit, et susceptibles d'évoluer en personnalités asociales 
ou antisociales, et l'on doit ainsi éviter les dépenses qu'ils entraîneraient... »

Konrad Lorenz, prix Nobel de médecine en 1974, écrivait également à cette époque : "Toute tentative de remise en état des éléments qui ont perdu leur relation avec la globalité est donc sans espoir. Par chance, leur élimination est plus facile pour le "médecin du peuple" et moins dangereuse pour l'organisme de la collectivité que l'opération pratiquée par le chirurgien sur le corps d'un individu. La grande difficulté technique réside dans leur détection ». K. Lorenz, "Les troubles du comportement spécifique à l'espèce provoqués par la domestication", in Zeitschrift F. angew. Psych. u. Charackterkunde, 1940, $59,2$.

(30) R. Lafon,

"Recherche sur les critères d'inadaptation", Revue de droit sanitaire et social, 1971, 28.

(31) En 1904, son cou- les "prédélinquants", les "délinquants " et en englobant, dans certains cas, les criminels et les chômeurs.

Les raisons invoquées a posteriori (en 1971) par le président de cette commission, le psychiatre Robert Lafon, pour rendre compte de ce choix sont les suivantes:

"Nous avons cherché une terminologie générale, dans un souci de simplification, de brièveté et de délicatesse, car les termes utilisés étaient insuffisants ou choquants ». ${ }^{(30)}$

Il me paraît toutefois nécessaire de rappeler que cette nouvelle catégorie d'usage du terme "inadapté" constitue à ce moment une métaphore active et un euphémisme; le terme est alors directement référé à la théorie biologique de l'évolution des espèces de Darwin. ${ }^{(31)}$ Cette théorie, d'après laquelle la transformation des espèces est due essentiellement à l'élimination des inadaptés par sélection naturelle, est à cette époque couramment utilisée par les nazis comme justification de leur politique raciale. Aujourd'hui, cette métaphore est éteinte; c'est un mot comme un autre, un mot anodin entré dans le langage courant. En 1943, ce n'était pas le cas.

Les séances du Conseil technique, tenues les 26 et 27 juin 1944, semblent avoir été dominées par l'urgence de faire aboutir ces travaux. Urgence sur les causes de laquelle il y a lieu de s'interroger, alors que les alliés ont débarqué en Normandie depuis trois semaines? Contrairement aux précédents, le procès-verbal de ces séances est squelettique.

Nous y apprenons cependant qu'il " avait été décidé de ne pas différer la réalisation des études entreprises, - études dont les conclusions doivent être à la base du règlement d'administration publique, sans lequel la prochaine loi sur l'Enfance déficiente ne pourrait être appliquée dans le plus bref délai soubaitable ".

Et Heuyer pense nécessaire de préciser que ces travaux constituent " une ouvre qui n'a aucun caractère politique, qui a un but uniquement social ".

Nous y apprenons également que "la discussion sur la standardisation de la nomenclature et les techniques d'examen a été remise à une date ultérieure " et qu'il « en fut de même du rapport établi par le Docteur Dublineau, en collaboration avec Mlle Meizex, et qui avait été confié à cette dernière sur la "définition et organisation des établissements de rééducation" ". Nous y apprenons que les travaux du conseil vont se poursuivre en comité restreint avec pour tâche : 
- de réunir les dossiers concernant l'observation des enfants dans les centres de triage;

- de "standardiser un modèle de fiche de synthèse, susceptible de suivre l'enfant dans ses transferts »;

- d'« instaurer, selon le modèle qu'a proposéle Docteur Fouquet, une fiche perforée universelle, destinée aux statistiques".

"En conséquence, les travaux de ce comité restreint seront communiqués par correspondance, dans le courant du mois d'août, aux membres $d u$ Conseil technique qui pourront s'y associer de loin."

Nous y apprenons aussi que "le matériel réuni restera à la disposition des spécialistes" au ministère.

Fort heureusement, à la date prévue (août 1944), Paris était libéré.

Mais le dispositif institutionnel issu des travaux du Conseil technique constitue un outil de pouvoir et de contrôle social auquel il sera difficile de renoncer.

Le communiste marseillais François Billoux, chargé au sein du Comité français de Libération nationale de prévoir les mesures à prendre en ce qui concerne la Santé et la Jeunesse, devient ministre de la Santé à la Libération. Il fera siennes les conclusions du Conseil technique de 1943 et décidera de la reconduction et de l'extension de la politique correspondante. Le "Plan Plaquevent", tombé dans l'oubli, ne sera, à ma connaissance, mentionné par aucun auteur depuis cette date.

\section{En guise de conclusion}

Le moment n'est pas venu, je crois, de tirer des conclusions; je n'ai fait qu'effleurer le problème et bien d'autres questions mériteraient d'être posées.

Il reste que vouloir comprendre ce que l'on récuse n'a rien de réjouissant; mais il faut reconnaître que le rejet dans l'inexprimé a quelque chose de paralysant.

Les travailleurs sociaux connaissent les conséquences que peuvent avoir sur la vie d'un être humain les zones d'ombre persistantes autour des circonstances de sa naissance ou les effets d'une paternité falsifiée. En serait-il de même en ce qui concerne un secteur professionnel?

sin Francis Galton inaugurait, à l'université de Londres, un enseignement national d'Eugénique, science dont l'objet consiste à «entraver la multiplication des inaptes, an lieu de les laisser venir au monde [...] et améliorer la race en favorisant la reproduction des plus aptes..."

Le Pr. Von Vershuer écrivait au Pr. Fischer, le 5 novembre $1937:$ : Il est toutefois important que notre politique raciale ait, $y$ compris sur la question juive, un fondement objectivement scientifique et reconnu par de larges couches de la population ». Cité in B. Muller-Hill, Tödliche Wissenschaft. Trad. française : Science nazie science de mort, Paris, Odile Jacob, 1989. Il existe cependant de bonnes raisons de penser que Hitler lui-même ne croyait pas au fondement biologique de sa politique raciale. Cf., op. cit, p. 95. 